DOI: http://dx.doi.org/10.19177/reen.v9e120163-25

http://portaldeperiodicos.unisul.br/index.php/EeN/index

\title{
BARREIRAS IMPOSTAS PELA CULTURA NA IMPLANTAÇÃO DE BALANCED SCORECARD EM UMA INDÚSTRIA DE ALIMENTOS
}

\section{BARRIERS IMPOSED BY CULTURE IN THE IMPLEMENTATION OF BALANCED SCORECARD IN A FOOD INDUSTRY}

\section{BARRERAS IMPUESTAS POR LA CULTURA EN LA IMPLANTACIÓN DE BALANCED SCORECARD \\ EN UNA INDUSTRIA DE ALIMENTOS}

Danilo Dias Pereira

Bacharel em Engenharia de Produção pela Universidade de Ribeirão Preto (UNIPI)

Endereço: Cidade Universitária, CEP: 05508-900. São Paulo, SP, Brasil

Telefone: (11) 3091-3116

E-mail: danilo.diaspereira1@gmail.com

Leonardo Augusto Amaral Terra

Doutor em Ciências no Programa de Administração de Organizações da Universidade de São Paulo (USP)

Endereço: Cidade Universitária, CEP: 05508-900. São Paulo, SP, Brasil

Telefone: (11) 3091-3116

E-mail: prof@leoterra.com.br

Artigo recebido em 20/02/2015. Revisado por pares em 03/08/2015. Reformulado em 23/02/2016. Recomendado para publicação em 23/02/2016, por Ademar Dutra (Editor Científico). Publicado em 30/04/2015. Avaliado pelo Sistema double blind review. 


\section{RESUMO}

O objetivo deste artigo é explorar o desenvolvimento de barreiras impostas pela cultura organizacional na implantação de Balanced Scorecards (BSC), através estudo em uma grande indústria de alimentos. $O$ artigo contempla a abordagem da cultura organizacional em uma visão sistêmica, suportada pela metodologia de P. Checkland - Soft Systems Methodology, com a criação de modelos para posterior análise das relações entre os atores. A principal barreira cultural identificada foi a falha no processo de transferência do conhecimento. Os autores propõem a utilização de reforços positivos nos operadores que detém o conhecimento, de modo a difundi-lo em toda a unidade.

Palavras-chave: Balanced Scorecard; Soft Systems Methodology; Cultura Organizacional.

\section{ABSTRACT}

The purpose of this article is to explore the development of organizational culture barriers imposed by the implementation of Balanced Scorecards (BSC) through a comparative study in a large food industry. The article considers the approach of organizational culture by a systemic view, supported by the methodology developed by Peter Checkland - Soft Systems Methodology (SSM), with the creation of models for subsequent analysis of the relationships among the actors. The main identified cultural barrier was the failure of the knowledge transfer process. The authors propose the use of positive reinforcement for operators who are knowledge holders in order to spread it throughout the unit.

Key words: Balanced Scorecard; Soft Systems Methodology; Organizational culture.

\section{RESUMEN}

El objetivo de este artículo es explorar el desarrollo de barreras impuestas por la cultura organizacional en la implantación de Balanced Scorecards (BSC), a través de estudio en una gran industria de alimentos. El artículo contempla el abordaje de la cultura organizacional en una mirada sistémica, soportada por la metodología de P. Checkland - Soft Systems Methodology, con la creación de modelos para posterior análisis de las relaciones entre los actores. La principal barrera cultural identificada fue el fracaso en el proceso de transferencia del conocimiento. Los autores proponen el uso de reforzamientos positivos para los operadores que detienen el conocimiento, de modo a difundirlo en toda la unidad.

Palabras-clave: Balanced Scorecard; Soft Systems Methodology; Cultura Organizacional. 


\section{INTRODUÇÃO}

Dada a relevância da estratégia no cenário organizacional mundial, as organizações que se projetam como líderes mundiais nos negócios onde atuam têm, como característica, entender os benefícios que a estratégia traz nos resultados em médio e longo prazo, de modo que passaram a utilizá-la como vantagem competitiva em seus negócios. A estratégia figura em todos os tipos de negócios, seja um serviço ou uma indústria, uma vez que cada empresa presente em uma indústria possui uma estratégia competitiva (PORTER, 2004).

A adoção de uma estratégia competitiva está diretamente relacionada com a mudança de mentalidade organizacional, como afirma Drucker (1999): "as novas realidades e suas demandas requerem uma reversão de políticas que funcionaram bem no último século e, ainda mais, mudança na mentalidade das organizações e das pessoas". A mudança citada por Drucker pode introduzir o conceito da operacionalização da estratégia organizacional, que pode ocorrer tanto nos níveis estratégicos como nos táticos, e nos níveis operacionais na organização. A operacionalização da estratégia figura não só em áreas administrativas, de modo que as operações industriais também estejam comtempladas em um conjunto hierárquico organizacional proposto. O conjunto hierárquico organizacional é uma resultante da operacionalização estratégica, como afirmam Mintzberg, Ahlstrand e Lampel (2010): “a operacionalização de estratégias dá origem a todo um conjunto de hierarquias, em diferentes níveis e com diferentes perspectivas de tempo". Com isso, a operacionalização da estratégia em indústrias passa a introduzir KPI (Key Performance Indicators), que vem a ser uma das principais ferramentas de mensuração de desempenho organizacional.

Com o passar dos anos, dois professores de Harvard (Kaplan e Norton), com o objetivo de inovar os métodos de mensuração de desempenho organizacional, desenvolveram, em 1992, a metodologia Balanced Scorecard (BSC) (KAPLAN; NORTON, 2004). Esta metodologia tornou-se referência mundial e é utilizada por diversas organizações, seja como pilar estratégico ou, ainda, como base para jornadas de melhoria contínua nas operações. O método BSC pode ser considerado a melhor opção para a realização dos desdobramentos dos objetivos industriais, pois é um conjunto de práticas que alinha pessoas e suas ações às prioridades do negócio, assegurando a execução confiável 
dessas ações. Todavia, caso seja utilizado em conjunto com ferramentas normalmente utilizadas em gestão da qualidade, pode alavancar o processo de melhoria contínua organizacional.

Os indicadores de desempenho do BSC promovem um link com a estratégia organizacional, de modo que a busca pela causa raiz dos problemas também esteja direcionada aos objetivos organizacionais. Ainda que a implantação do BSC seja de suma importância para as grandes organizações, sua eficiência como apoio à melhoria contínua depende de um processo de mudança de cultura organizacional. A cultura está relacionada diretamente aos comportamentos dos colaboradores de uma organização. Qualquer método adotado por uma organização que envolva a mudança de cultura possui uma longa curva de aprendizagem, e requer empenho de todos os envolvidos.

Diante dos argumentos expostos, e considerando os problemas culturais que uma organização pode enfrentar, este artigo tem como objetivo entender como se desenvolvem as barreiras impostas pela cultura organizacional na implantação de BSC na indústria.

\section{ESTRATÉGIA, BALANCED SCORECARD E PROCESSOS INTERNOS}

A estratégia organizacional pode ser definida como um conjunto de ações que uma organização executa com o intuito de alcançar os objetivos que defende e almeja em sua missão e visão, respectivamente, com a utilização dos valores organizacionais como diretrizes às ações que executará ao longo de sua existência (NEIS; PEREIRA, 2015). Para Mintzberg, Ahlstrand e Lampel (2010), a estratégia é uma coerência nos comportamentos organizacionais ao longo do tempo.

De maneira formal ou informal, as organizações, independentemente de sua classificação econômica, formulam objetivos que buscam trazer vantagens no futuro. Esta visão projetada do futuro pode ser definida como planejamento estratégico. A tomada de ações contempladas pelo planejamento estratégico organizacional é monitorada pelos resultados que, segundo Morales, Gonzales e Mendoza (2013), são avaliados de forma limitada, quando feitos apenas por indicadores financeiros e contábeis, tais como lucro no exercício, faturamento e endividamento. 
Nos últimos anos, as organizações iniciaram um processo de mudança na visão dos negócios, de forma que os mesmos sejam direcionados à agregação de valor aos clientes, mudanças de práticas, eficiência operacional e desenvolvimento de pessoas. Tal mudança na visão organizacional introduz uma nova maneira de formular objetivos e execução da estratégia no dia-a-dia, que é subsidiada pelo Balanced Scorecard (BSC). Criado por Kaplan e Norton (1997), O BSC é um método de mensuração estratégica com base em quatro perspectivas (Financeira; Clientes, Processos internos; Aprendizado e conhecimento) e, segundo Spessatto e Beuren (2013), o BSC obteve uma evolução desde sua criação, tornando-se um sistema gerencial essencial para obtenção de feedback sobre a estratégia. $\mathrm{O}$ feedback sobre a estratégia permite um mapeamento de issues organizacionais, e traz propriedade à tomada de decisão gerencial. Frezatti, Relvas e Junqueira (2010) afirmam que o BSC é iniciado com base na demanda por projeções de resultados. Da perspectiva de processos internos abordados pelo BSC, a manufatura possui grande relevância nos resultados organizacionais, pois é uma fonte de oportunidades de melhorias em eficiência operacional. Porém, os processos internos industriais podem variar em cada organização, bem como os objetivos e indicadores, e o desdobramento para gerenciamento dos processos internos industriais com base em BSC deve contemplar os seguintes indicadores listados no Quadro 1.

Quadro 1 - Desdobramento para gerenciamento de processos internos industriais

\begin{tabular}{|l|l|}
\hline Indicador & Descrição \\
\hline Produtividade & Confiabilidade (Performance e eficiência) \\
\hline Qualidade & Conformidade da produção \\
\hline Custos & $\begin{array}{l}\text { Custo de conversão, produtos não conforme, } \\
\text { retrabalho e variações de utilização }\end{array}$ \\
\hline Entrega de Produção & Disponibilidade e nível de serviço \\
\hline Segurança & Acidentes de trabalho \\
\hline
\end{tabular}




\begin{tabular}{|c|c|}
\hline Saúde e Meio ambiente & Consumo de recursos naturais (água e energia) \\
\hline Moral & Absenteísmo \\
\hline
\end{tabular}

Fonte: Adaptado de Slack, Chambers e Johnston (2009).

Os indicadores devem possuir um desdobramento desde o comitê executivo (liderança industrial) até os grupos de trabalho, utilizando a estratificação pela abrangência (Indicadores de Fim) e relação causa-efeito (Indicadores de Meio). A estratificação dos indicadores é essencial para o alcance das metas estabelecidas e deve refletir uma consolidação para avaliação do comitê executivo. Segundo Barreto et al. (2013), pelo fato de possuírem um viés quantificável, as metas contempladas pelo BSC são tidas como um instrumento importante de gerenciamento de esforço de implantação e desempenho, o que permite afirmar que podem ser consideradas como um dos fatores críticos da implantação da estratégia. Contudo, torna-se importante ressaltar que, tal como Silva (2003, p.5) também afirma, "a solução para uma efetiva implantação de estratégias vai além do emprego de um método ou de outro".

O processo de melhoria contínua está relacionado a várias práticas gerenciais, que podem ser definidas como perenes, de modo que a organização sempre esteja engajada em melhorar suas operações e processos. Furlan Jr, Cardos e Chaves (2010) propõem que a melhoria contínua em uma organização seja como um ciclo virtuoso. Um dos atributos que contemplam a melhoria contínua é o empowerment que, na visão de Shiri, Soltani e Abadi (2013), é uma combinação de técnicas motivacionais buscando aumentar o nível de cooperação do pessoal para melhorar seu desempenho. Em uma indústria, o empowerment está relacionado à autonomia dos colaboradores na tomada de decisão.

Considerando uma organização onde os KPI propostos pelo BSC já são considerados uma realidade, os executivos passam a entender que grande parte das ações que irão atacar a causa raiz dos problemas está dentro do raio de alcance dos colaboradores presentes nas operações, de modo que adotem uma estratégia combinatória entre o BSC e métodos de grande sucesso na área de gestão da qualidade, tais como $5 \mathrm{~W} 2 \mathrm{H}$, Diagrama de Pareto e Diagrama de Causa-Efeito (Ishikawa), utilizando o empowerment como agente de engajamento para a aplicação dos métodos. 
Morales, Gonzales e Mendoza (2013) definem o empowerment como uma experiência educacional participativa, visando a aumentar as habilidades gerenciais e dando ferramentas eficazes, que são essenciais para permitir que as pessoas mantenham um equilíbrio do estilo de vida e ter, como resultado, um ambiente de trabalho mais harmonioso.

A estratégia combinatória somada ao empowerment é uma poderosa vantagem competitiva que uma organização pode entregar, e vem sendo utilizada com maior frequência nos últimos anos. Conforme a afirmação de Bon e Mustafa (2014), a participação dos funcionários no processo aumenta sua lealdade, comprometimento e criatividade, que apoiam a inovação. Contudo, a vantagem competitiva só é entregue quando há engajamento em todos os níveis organizacionais, ou seja, do mesmo modo que os executivos e gerentes buscam a melhoria contínua avaliando os indicadores do BSC, os operadores também necessitam executar as mesmas práticas em suas atividades diárias.

O BSC pode ser considerado como o passo inicial para o engajamento na jornada de melhoria contínua das operações como um todo. O engajamento e o comprometimento dos operadores em muitas indústrias são fatores de grande desafio para os executivos, uma vez que a cultura dos colaboradores e a própria cultura organizacional, criada antes da implantação do BSC, não seguem a mesma linha de raciocínio.

\section{CULTURA ORGANIZACIONAL E PROCESSO DE MUDANÇA}

O termo cultura possui inúmeras definições e está diretamente relacionado à maneira de execução de quaisquer atividades executadas por pessoas, sejam em grupo ou individualmente, bem como nas organizações. No que tange às organizações, o modo como a empresa executa suas atividades, diferenciando-as das demais organizações, pode ser denominado como cultura (MINTZBERG; AHLSTRAND; LAMPEL, 2010). Sobral e Peci (2008) defendem a ideia de que, mesmo com a semelhança de fatores externos nas organizações, a influência real é dada conforme a singularidade da própria organização. Freitas (1991) afirma que os elementos da cultura organizacional mais citados são: Valores; Crenças e pressupostos; Ritos, rituais e cerimonias; Histórias e mitos; Tabus; Heróis; Normas; e Processo de comunicação. Tais elementos são capazes de fornecer uma interpretação para os membros da organização. 
Desde o início de sua aplicação na administração, na década de 80, com o conhecido modelo japonês de Liker e David (2005), atualmente a cultura pode ser considerada como um sistema complexo que, em muitas situações, são desconhecidos por gestores (BERNSTORFF; COSTA; GIRARDI, 2003). Terra e Passador (2014) vão além, e afirmam que, dada a complexidade, é imprescindível entender os elementos necessários para a gestão das relações internas e externas nas organizações e, com base nesta afirmação, é possível assegurar que os gestores possuem papel fundamental nesta governança. Uma vez que os gestores não possuam o conhecimento da cultura de suas organizações, o desempenho organizacional, conforme afirmação de Barreto et al. (2013), pode ser comprometido, pois o desempenho da organização é fruto dos valores e crenças compartilhados por seus membros e pelo compromisso por eles assumido.

O papel dos gestores também é evidenciado pela definição do conceito de cultura organizacional defendido por Fleury (2009), que relaciona à busca de vantagem competitiva através da reprodução de práticas de gestão oriundas da multinacionalização, e sua baixa eficácia às dificuldades enfrentadas em países estrangeiros pelos gestores. Santos e Gonçalves (2010) afirmam que as organizações se adaptam à evolução das sociedades, e essa evolução origina uma mudança dos valores culturais. A mudança dos valores organizacionais também é chamada de mudança de cultura organizacional. Esta se inicia com as transformações estratégicas que, por sua vez, também podem ser influenciadas pela cultura (OLIVEIRA; TERRA, 2013), como exposto por Mintzberg, Ahlstrand e Lampel (2010, p.190): “a cultura influencia o estilo de pensar favorecido numa organização, assim como seu uso de análise". O processo de mudança de cultura deve ser inserido em uma reformulação estratégica com o envolvimento dos gerentes, ainda que os mesmos possam ser influenciados, conforme dito por Silva (2003, p.100) “os gerentes não só podem esquecer alguns detalhes importantes, como também deixar de elaborar uma estratégia principal para a mudança planejada".

O desenvolvimento dos gerentes e membros da organização para o processo de mudança de cultura é considerado, por Neiva e Paz (2012), uma importante ferramenta para o sucesso, uma vez que a preparação constrói uma base para atitudes de aceitação em relação aos programas de mudança, diminuindo o desgaste proporcionado pelos mesmos. No entanto, várias teorias comprovam que o processo de mudança apresenta uma curva de 
aprendizagem que, por sua vez, possui o tempo como um eixo horizontal. Para alcançar a eficácia do processo de mudança de cultura, Powell (1995) defende a ideia que as organizações precisam de intuição, símbolos e arte que ocorrem no seu dia-a-dia. Esta afirmação introduz o conceito de flexibilidade para a organização que almeja a melhoria contínua.

Ao longo da evolução e sustentabilidade do processo de mudança de cultura, o nível de engajamento dos colaboradores passa a ser mensurado, e os executivos criam uma base sólida para a tomada de medidas estratégicas relacionadas à turnover, conforme exposto na afirmação de Wood Jr, Curado e Campos (1994, p. 65): "pessoas que não aceitam novos padrões devem ser removidas para evitar racionalizações inapropriadas sobre o processo". Wood Jr, Curado e Campos (1994) ainda afirmam que a mudança do sistema normativo contempla a mudança de cultura, e os novos padrões implícitos ou explícitos de punição e recompensa são considerados um complemento institucional.

A mudança de cultura estará presente em toda implantação de processos em uma organização, o que contempla também o BSC. Segundo a visão de Domingues e Bueno (2004), em uma implantação de BSC, tanto a estrutura organizacional como as pessoas envolvidas na mudança devem ser consideradas pela influência que exercerão sobre estes processos e, muitas vezes, a influência poderá se apresentar na forma de resistência à mudança. As variáveis culturais podem ser fatores determinantes no sucesso de uma implantação, de modo que este artigo permita entender quais barreiras são criadas na implantação de BSC.

\section{MÉTODO DE PESQUISA}

A proposta de pesquisa contemplada por este artigo é exploratória. Conforme a afirmação de Gil (2002), as pesquisas exploratórias têm como objetivo aumentar a familiaridade com o problema, com o intuito de torná-lo mais explícito, bem como constituir hipóteses. A abordagem utilizada na pesquisa a define como qualitativa, pois, de acordo com o mesmo autor, a interpretação dos fenomenos, bem como a atribuição dos significados a contemplam e, como não pode ser traduzida em números, a pesquisa qualitativa não requer o uso de técnicas e métodos estatísticos. 
O campo escolhido para a aplicação do método de pesquisa proposto é uma indústria de alimentos de grande porte e, com o intutio de analisar o problema com uma abordagem sistêmica, neste artigo é contemplada pela metodologia proposta por Peter Checkland, Soft Systems Methodology (SSM), que será aborada nos próximos tópicos. O objetivo da pesquisa deste artigo limita-se na sugestão de critérios para a elaboração de ações que melhorem o engajamento das equipes para auxílio na busca pela causa raiz dos problemas dispostos no dia-a-dia da organização estudada.

\section{a. Soft Systems Methodology}

Para Checkland (2000), a SSM pode ser definida como uma metodologia que analisa problemas relacionados a questões comportamentais pouco estruturados dentro da perspectiva organizacional. Nela, o foco em um objetivo unitário é caso especial para debater múltiplas percepções, procedendo com base em acomodações entre diferentes interesses. Ferolla (2013,p.88) destaca que a SSM é frenquentemente utilizada nos "estudos sobre realidades complexas e a aderência às características de complexidade inerente dos sistemas políticos".

Segundo Cezarino, Liboni e Martinelli (2005), a diferneça entre SSM e as demais metodologias é sua capacidade de diagnóstico de dificuldades e, através da perspectiva sistêmica, contribuir com a real fonte do problema.

O modelo proposto por Checkland para aplicação da SSM contempla o ciclo de descoberta, investigação e aprendizado, conforme exposto na Figura 1.

Figura 1 - O ciclo de descoberta, investigação e aprendizado da SSM 


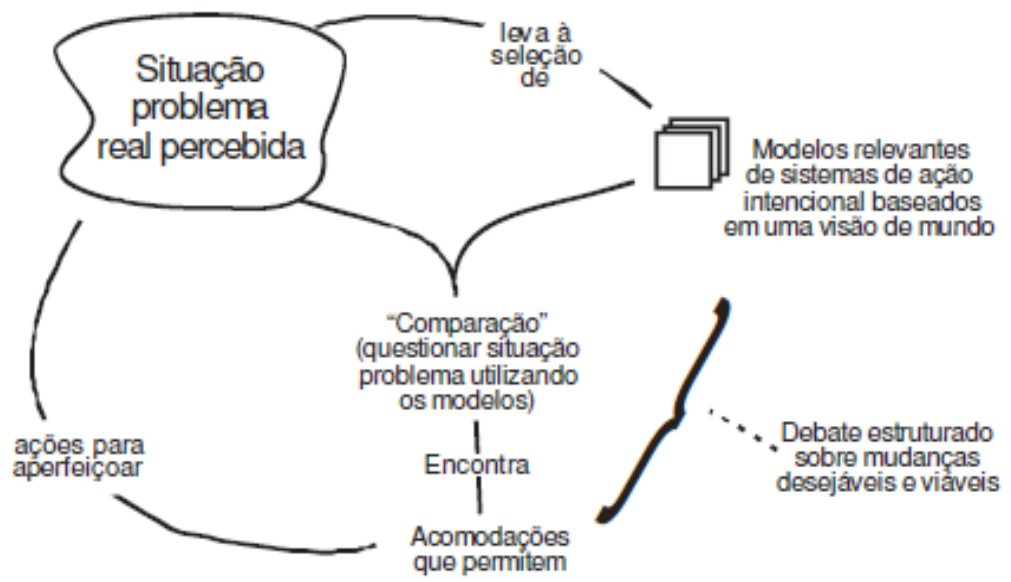

Fonte: Ferolla (2013) - traduzido e adaptado de Checkland (2000, p. 88).

Os estágios da execução da SSM contemplados no ciclo proposto na Figura 1 e que serão realizados no decorrer da pesquisa estão listados no Quadro 2.

Quadro 2 - Estágios para a execução da SSM

\begin{tabular}{|l|l|l|}
\hline Estágios & Descrição \\
\hline $\begin{array}{l}\text { problema - Mapeamento do } \\
\text { perspectiva prática }\end{array}$ & $\begin{array}{l}\text { Representação gráfica dos pesquisadores e entrevistados - } \\
\text { evidenciação dos entendimentos individuais do problema. } \\
\text { Identifica os autores e as relações estabelecidas entre eles. }\end{array}$ \\
\hline $\begin{array}{l}\text { conceituais Modelos } \\
\text { perspectiva teórica }\end{array}$ & $\begin{array}{l}\text { Os modelos conceituais que contemplam as atividades- } \\
\text { chave do sistema principal. Identifica os autores e as } \\
\text { relações estabelecidas entre eles. }\end{array}$ \\
\hline 3 e 4 - CATWOE & $\begin{array}{l}\text { Construção do modelo com identificação dos sistemas } \\
\text { relevantes para análise descritos nos estágios } 1 \text { e 2, } \\
\text { conhecendo a natureza } \\
\text { Conjuntos de elementos: C (Customers), A (Actors), W } \\
\text { (Weltanschauung), O (Owner) e E (Environmental } \\
\text { contraints). }\end{array}$ \\
\hline
\end{tabular}

Fonte: Adaptado de (Ferolla, 2013).

Revista Eletrônica de Estratégia \& Negócios, Florianópolis, v.9, n.1, jan./abr. 2016. 


\section{b. Contextualização do campo de pesquisa}

O campo escolhido para aplicação do método de pesquisa baseado em SSM é uma indústria de alimentos de grande porte, que possui dezoito unidades produtivas no Brasil e uma sede corporativa na cidade de São Paulo - SP. A planta de estudo é uma unidade presente na cidade de Ribeirão Preto, onde trabalham cerca de trezentos e cinquenta colaboradores regulares, divididos em três turnos de operação, em seis dias por semana. A indústria possui três linhas de produção e oito de envase, que trabalham em produção por processos.

Recentemente a organização lançou uma jornada rumo à excelência em suas operações manufatureiras, de modo a implantar a missão denominada Rumo ao Zero, difundida nas dimensões: Meio Ambiente (Zero impacto), Segurança (Zero acidentes), Entrega (Zero Ruptura), Qualidade (Zero defeitos) e Custo (Zero perda). Para suportar a busca pelo zero defendida pela organização, foram introduzidas diversas ferramentas amplamente conhecidas na área acadêmica e abordada pelos estudos de administração da produção, tais como TPM (Total Productive Maintenance), 5S, Six Sigma e demais ferramentas de gestão da qualidade (Diagrama de Pareto, Diagrama de Ishikawa e 5 Porquês). Os BSC foram escolhidos para suportar os KPI (Key Performance Indicators) de todas as operações, seguindo o conceito de fundação em um modelo desenvolvido em pilares, tornando-se um agente de feedback para a Diretoria e apoio para a tomada de ações que buscam solucionar a causa-raiz dos problemas.

Foram implantadas reuniões operacionais diárias, onde os operadores dos processos possuem um tempo em sua passagem de turno dedicado à atualização do plano de produção para o próximo turno, ao apontamento de issues relacionados à segurança ocorridos no turno anterior, mas, principalmente, à avaliação dos BSC e criação de ações que buscam identificar a causa-raiz dos desvios apresentados, sejam eles operacionais (de solução dentro da própria operação) ou técnicos (necessária intervenção do setor de manutenção). O plano de ações gerado diariamente nas reuniões operacionais é considerado, pela organização, o principal feedback da aderência dos colaboradores ao programa de melhoria contínua, uma vez que através dele se mantêm registradas todas as buscas realizadas para eliminação dos problemas. 


\section{c. Ferramentas e Procedimentos de coleta de dados}

A coleta de dados necessários para avaliação do problema proposto foi suportada por uma pesquisa participante. Este tipo de pesquisa, conforme definição de Gil (2002), possui caráter consideravelmente comprometido com a minimização da relação entre dirigentes e dirigidos, de modo que seja voltada para investigação em grupos considerados desfavorecidos, tais como operadores. Um dos pesquisadores conduziu a pesquisa participante com o papel de coaching nas reuniões operacionais de BSC, com o intuito de observar o comportamento das equipes e dos colaboradores individualmente, além de tomar notas de todos os pontos relevantes apontados durante a apresentação dos KPI. A pesquisa participante foi definida como instrumento base para a criação da modelagem da SSM, onde há representação gráfica que identifica as relações entre todos os atores e suas relações.

A pesquisa em campo feita por um dos pesquisadores avaliou sete das dez trocas de turno da fábrica, em dois turnos de produção, totalizando quatorze avaliações. Nelas os pontos avaliados foram: preparação para a troca de turno, conhecimento dos problemas no turno, avaliação dos KPI definidos e qualidade do plano de ação. O fluxo da reunião permitiu a criação de uma ferramenta com perguntas predefinidas onde o pesquisador pôde avaliar e, com base nos resultados para cada pergunta ( 2 pontos para positivos, 1 ponto para parcialmente positivo e 0 pontos para negativos), definir o percentual de aproveitamento de cada um dos times.

Na ferramenta de avaliação há um campo disponível para o pesquisador apontar o tempo de companhia de cada um dos dois operadores participantes, uma vez que essa informação será a base para comparação do engajamento.

\section{APRESENTAÇÃO DE RESULTADOS}

A Figura 2 representa o modelo de rotina diária das atividades que toda a operação da fábrica executa, desde as reuniões com o comitê executivo (gerentes), passando pelo comitê local (mão de obra de apoio e coordenadores), até chegar aos operadores (trocas de 
turno). As reuniões avaliam os KPI divididos em Performance, Processo e Atividades, com um gatilho automático para a geração de ações. O plano de ação é baseado em $5 W 1 \mathrm{H}$ e pode ser uma ação de curto prazo, ou seja, enxergar o problema no local onde realmente acontece, ou ações de longo prazo, que utilizam métodos resolução de problemas (Brainstorming, Projetos Six Sigma DMAIC e Diagrama de Ishikawa). O reflexo desse plano de ação é indicado pela seta vermelha. As setas azuis representam a ligação dos elementos da Figura.

Figura 2 - Modelo de gestão da rotina de operações da indústria de alimentos

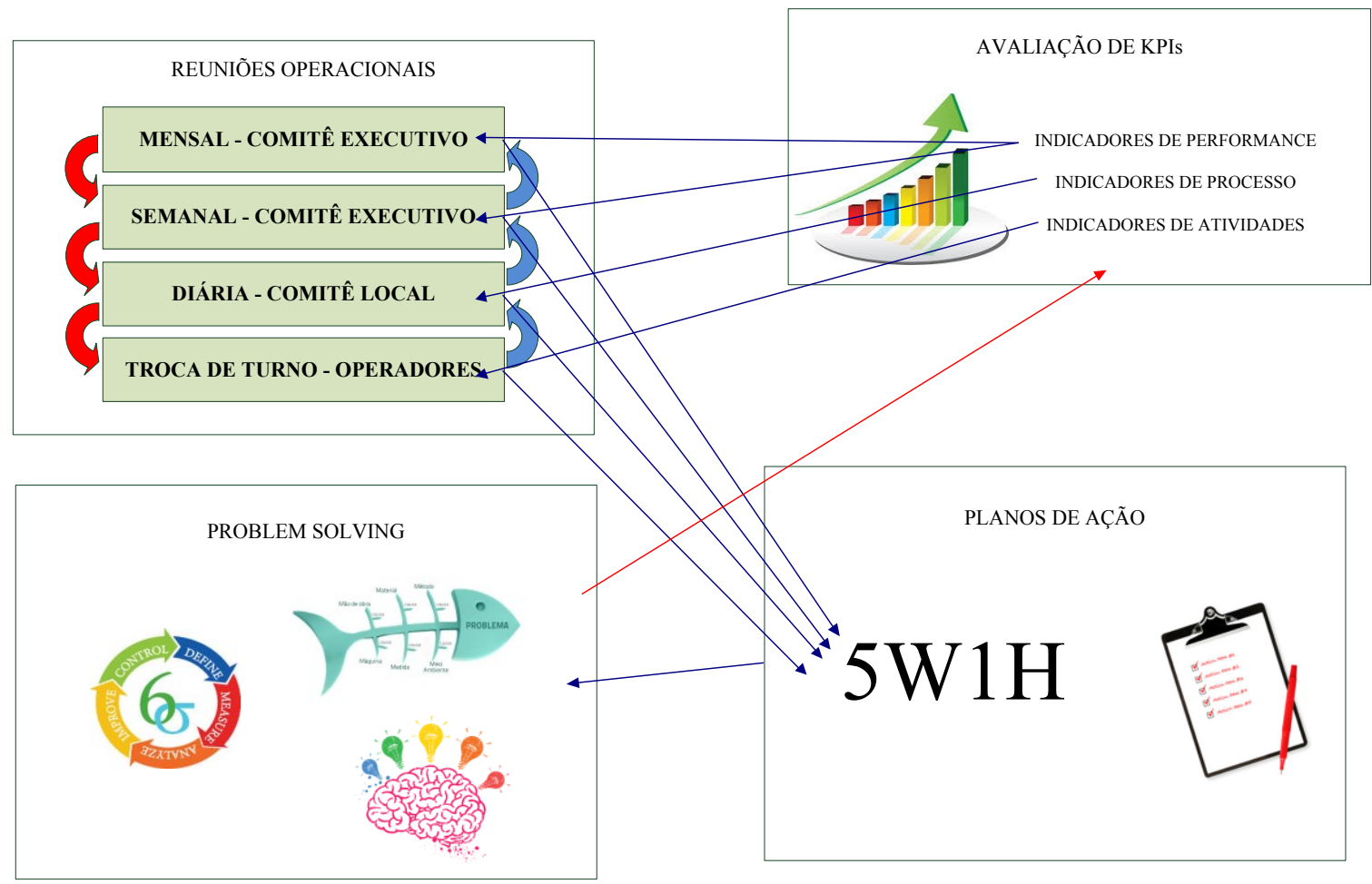

Fonte: Elaborada pelos autores (2015).

Os resultados das avaliações durante as reuniões operacionais de troca de turno explicitaram diversas dificuldades que os times enfrentam. Em 78,6\% das avaliações, foram penalizadas as ações levantadas pelos operadores para a tratativa de problemas, ou seja, as ações foram corretivas, resolviam o problema de imediato, mas não buscavam atacar a causa raiz dos problemas. Um dos pesquisadores identificou que, em cerca de $55 \%$ destas reuniões penalizadas, apenas um dos participantes da reunião tinha conhecimento técnico para resolver o problema e, além de não haver geração de uma ação para a causa-raiz do problema, o conhecimento não foi compartilhado com o grupo.

Revista Eletrônica de Estratégia \& Negócios, Florianópolis, v.9, n.1, jan./abr. 2016. 
O segundo ponto que apresentou $92,8 \%$ de resultados negativos nas reuniões está relacionado à geração de padrões para tratamentos de problemas futuros. Ainda que impactados pela falta de ações que buscam a causa-raiz dos problemas, a correção imediata ou contenção do problema não foi traduzida em procedimento para que todos do time fossem treinados em tal resolução. A falta de compartilhamento entre o time possibilitou a presença de problemas desconhecidos por colaboradores recém-contratados.

Os resultados positivos obtidos foram atribuídos a dois times que executaram a troca de turno. O pesquisador avaliou que ambos os times correspondem a linhas novas, que iniciaram suas atividades em outubro de 2013, cerca de três anos após a implantação dos BSC na unidade produtiva. Todos os colaboradores foram contratados para as linhas novas; contudo, sua base de treinamentos é superior, quando comparada aos demais colaboradores.

\subsection{CONSTRUÇÃO DO CATWOE ENRIQUECIDO}

\section{i. $\quad$ - Customers}

No mapeamento sistêmico da fábrica de alimentos, os clientes do processo de execução dos BSC na operação são os operadores que recebem o turno em uma reunião operacional, pois necessitam do conhecimento adquirido no turno anterior, bem como os resultados obtidos, resultados que, por sua vez, serão direcionadores das ações que o time tomará. Como características necessárias aos clientes destacam-se: Conhecimento técnico de sua operação; Conhecimento nas principais ferramentas relacionadas à busca da causaraiz, tais como Brainstorming, Diagrama de 5 Por quês, Diagrama de Ishikawa, e Elaboração de plano de ação com base em 5W1H.

Os pesquisadores identificaram falta de criticidade dos clientes ao receber um turno de produção, situações onde houve problemas de outros turnos que não tiveram a tratativa foram aceitas pelos clientes sem quaisquer questionamentos, e quando questionadas durante a execução do seu turno de produção, ainda não possuíam resposta para o problema. 


\section{ii. A-Actors}

Os atores, neste modelo sistêmico, são os operadores que entregam o turno aos próximos operadores, que são os responsáveis pela atualização dos BSC, pelo feedback dos acontecimentos do turno aos supervisores, bem como o início da aplicação de ferramentas de qualidade para resolução de eventuais problemas. Assim como para os clientes, entre as características necessárias aos atores destacam-se: Conhecimento técnico de sua operação; Conhecimento nas principais ferramentas relacionadas à busca da causa-raiz, tais como Brainstorming, Diagrama de 5 Por quês, Diagrama de Ishikawa, e Elaboração de plano de ação com base em 5W1H.

Nota-se uma forte resistência dos atores na busca pela causa-raiz dos problemas. As avaliações das reuniões permitiram ao pesquisador observar a preferência dos atores em resolver o problema de imediato, ao invés de aplicar as ferramentas que a organização oferece.

\section{iii. T - Transformation Process}

O processo de transformação do sistema está focado na operacionalização da estratégia, onde o ator deve avaliar seus inputs, ou seja, KPI e práticas relacionadas ao turno em questão, levantar plano de ação que busque eliminar a causa-raiz do problema. Este processo de transformação trará os seguintes outputs: resultado, padronização e compartilhamento de conhecimentos.

Na organização, este fluxo é um modelo definido pela matriz internacional e promove o alinhamento entre todas as unidades em diferentes mercados. Contudo, como o cenário de melhoria contínua na organização está mundialmente em fase de aprendizagem e crescimento, o mercado brasileiro, que foi o pioneiro na implantação do modelo, identificou diversas melhorias e casos de sucesso que podem auxiliar o fluxo de reuniões, mas dependem de uma aprovação em nível corporativo e, como o processo de aprovação demanda tempo para avaliação em nível mundial, o modelo pode ser um fator que reduz a velocidade de crescimento e aprendizagem destacada pelos BSC. 


\section{iv. W-Weltanschauung}

A visão de mundo deste sistema está diretamente relacionada com a necessidade da organização entregar vantagem competitiva, considerando as dimensões de Rumo ao zero. Esta visão desencadeou uma série de mudanças no ambiente corporativo, que não se limitam simplesmente a uma forma de executar determinada atividade, mas em uma complexa mudança cultural, que foi apresentada ao mundo corporativo através do Sistema Toyota de Produção.

\section{v. $\quad 0$-Owners}

Os owners apresentados neste modelo sistêmico são os superiores imediatos dos operadores, estes são os supervisores de turno responsáveis por toda a gestão operacional da organização. Somados aos supervisores, os coordenadores de processo e o gerente de fabricação também exercem essa função-modelo, uma vez que são os responsáveis oficiais do setor de fabricação.

Nas avaliações dos pesquisadores, nenhuma das reuniões operacionais teve o acompanhamento dos owners que, em diversas situações, podem ajudar o time como suporte técnico ou, ainda, como link com outras áreas.

\section{vi. E-Environment}

O sistema proposto está relacionado à cadeia direta de fabricação dos SKU Stock Keeping Unit (SKU) da fábrica, ou seja, nele estão considerados apenas os processos e operações onde se concentram a mão de obra direta e os recursos gargalos do processo produto. Embora a missão Rumo ao zero da organização não se limite ao ambiente produtivo, mas a toda a cadeia de suprimentos, a participação das áreas de suporte teria impacto no modelo apenas se a organização estivesse com maturidade suficiente na resolução de problemas de baixa complexidade, que não envolvem questões estatísticas complexas e investimentos. 


\section{b. Figura rica}

A figura rica é uma representação gráfica livre sugerida pela SSM que contempla todos os atores envolvidos no cenário e suas relações no ambiente (FEROLLA, 2013). Segundo Rose (1997), a figura rica contém, normalmente, descrições de situações problemáticas locais e não tem explícita base teórica, e apresenta, como objetivo, obter caraterísticas abstratas e domínio do problema.

A Figura 3 representa a figura rica da indústria de alimentos, onde os atores e as principais ferramentas do modelo estão presentes. As relações que os mesmos devem possuir no modelo teórico estão representadas pelas setas azuis, e as entregas e contribuições que cada ator ou ferramenta proporciona ao modelo estão escritas em vermelho. As setas verdes simbolizam as relações que realmente ocorrem no ambiente de manufatura. Não há uma relação ou ator inicial para interpretação da figura rica. Optamos por exemplificar o Operador, ator central da figura rica, bem como suas relações. É correto afirmar que o mesmo deve avaliar o que os BSC estão dizendo e gerar um plano de ação. A ação deve utilizar ferramentas de resolução de problemas, e a correta aplicação trará conhecimento para o Operador, resultados que serão visualizados nos BSC e padronização, para que a Equipe de treinamento possa estruturar a capacitação de novos operadores.

Contudo, o cenário real se limita e restringe algumas relações propostas. Os operadores avaliam os BCS como necessários, mas geram planos de ação que não utilizam as ferramentas de melhoria contínua, e a resultante da ação, apesar de trazer resultado ao BSC, não pode estar diretamente relacionada à causa-raiz do problema, de modo a não ter mitigada a probabilidade de um evento similar acontecer. Em contrapartida, o conhecimento desta ação fica limitado ao operador executante, e não permite a atuação do time de treinamentos, que seria o agente de multiplicação do conhecimento na unidade.

Figura 3 - Figura rica da indústria de alimentos 


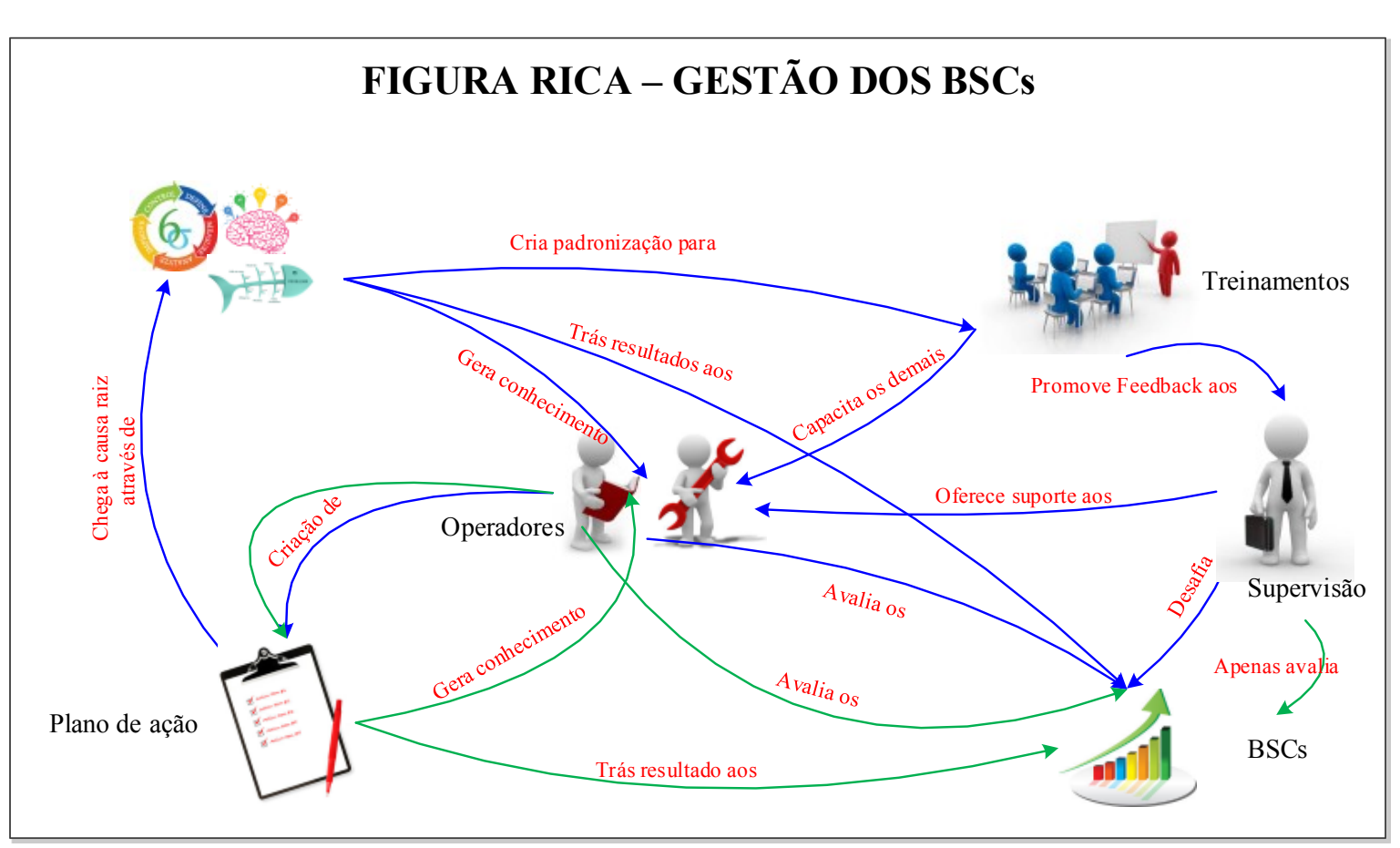

Fonte: Elaborada pelos autores (2015).

\section{i. Identificação de barreiras}

A falta de suporte dos owners no cotidiano dos operadores foi identificada como a primeira barreira cultural que a implantação de BSC proporciona. Os líderes diretos não foram preparados para realização de seções de coaching com os operadores, de modo a orientá-los na busca de alternativas que eliminem a causa-raiz dos problemas.

A segunda e principal barreira identificada pelos pesquisadores está relacionada à falta de transferência de conhecimento, onde os operadores deixam de criar novos padrões para a execução das atividades, que, na visão dos pesquisadores, cria um ciclo que causa uma interferência em todo o plano de treinamento para novos colaboradores e reciclagens de conhecimento promovidas pela unidade. O ciclo está representado pela Figura 4.

Figura 4 - Ciclo de conhecimento operacional 


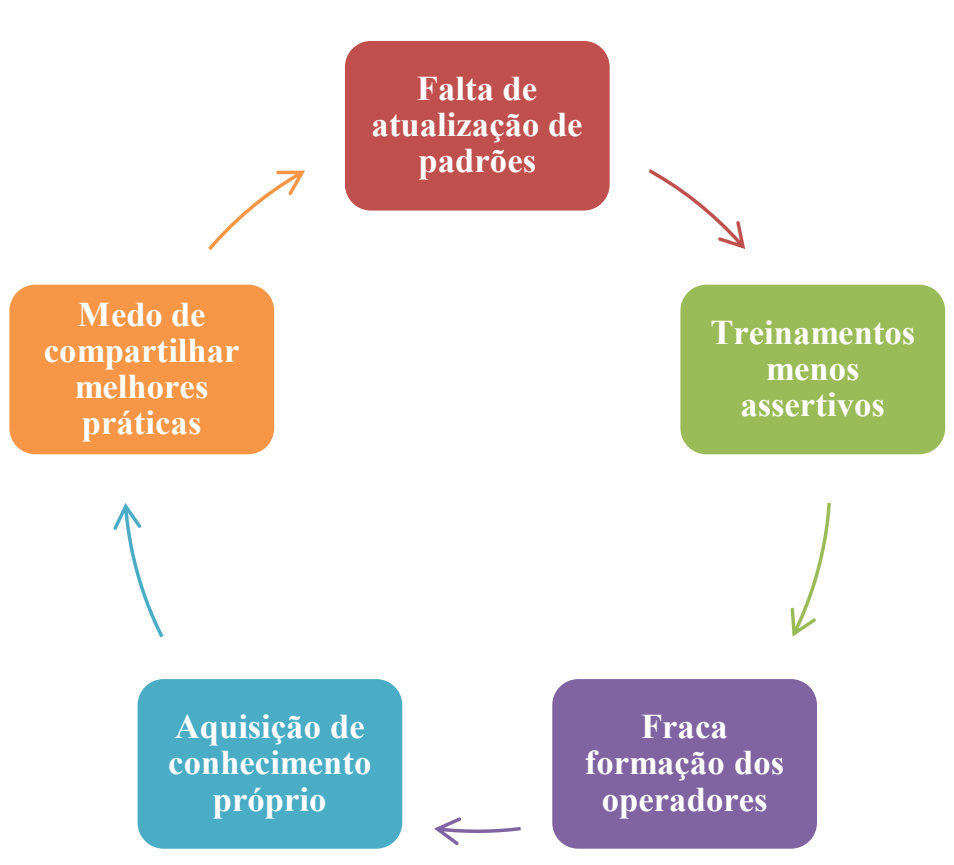

Fonte: Elaborada pelos autores (2015).

\section{CONSIDERAÇÕES FINAIS}

A construção deste artigo teve como objetivo entender quais são as barreiras impostas pela cultura organizacional na implantação de BSC em uma indústria de alimentos. Os autores buscaram avaliar o problema com a metodologia sistêmica SSM, que realiza o mapeamento do problema com atores, bem como a relação que os mesmos estabelecem entre si e em conjunto com as ferramentas.

$\mathrm{Na}$ visão dos pesquisadores, os primeiros treinamentos criados para capacitar os supervisores e operadores não foram efetivos, de modo que o ciclo apresentado na Figura 4 tornou-se presente no cotidiano das operações, ou seja, os operadores possuem fraca formação e adquirem o conhecimento no decorrer da execução de suas atividades. Esse conhecimento adquirido passa a fazer parte da identidade dos operadores, e o tornam indisponível aos demais envolvidos no processo, sejam outros operadores ou, ainda, supervisores e outros interessados no processo produtivo. A falta de compartilhamento do conhecimento faz com que os padrões não sejam atualizados e estes, que são utilizados para treinamento dos novos colaboradores, não refletem a realidade do processo.

Para quebrar o desenvolvimento destas barreiras, os autores propõem que a organização selecione operadores que detêm melhores conhecimentos em suas áreas de 
atuação e estimule-os à criação de padrões utilizando reforços positivos de comportamento operante. Na visão de Skinner (1982), aumenta a probabilidade de um comportamento ocorrer novamente, de modo a difundir esta prática nos formadores de opinião, bem como melhorar a integração e a funcionalidade de seus treinamentos.

O objetivo da pesquisa deste artigo limitou-se à sugestão de critérios para a elaboração de ações que melhorem o engajamento das equipes para auxílio na busca pela causa-raiz dos problemas dispostos no dia-a-dia da organização estudada. Os autores entendem que existem oportunidades de desenvolvimento de novas pesquisas que compreendam outras barreiras que a cultura cria na implantação de BSC.

\section{REFERÊNCIAS}

BARRETO, L. M. T. S.; KISHORE, A.; REIS, G. G.; BAPTISTA, L. L.; MEDEIROS, C. A. F. Cultura organizacional e liderança: uma relação possível?. Revista de Administração, v. 48, n. 1, p. 34-52, 2013.

BERNSTORFF, B. W.; COSTA, A. M.; GIRARDI, D. A Contribuição Do Balanced Scorecardcomo Instrumento De Gestão Organizacional. Revista de Administração, v. 2, n. 3, p. 22, 2003.

BON, A. T.; MUSTAFA, E. M. A. Impact of Total Quality Management-Based People Management Practices on Administrative Innovation In Service Smes. Middle-East Journal of Scientific Research, v. 19, n. 9, p. 1162-1168, 2014. Disponível em: < http://www.idosi.org/mejsr/mejsr19(9)14/7.pdf >. Acesso em: 20 mai. 2015.

CEZARINO, O. L.; LIBONI, L.; MARTINELLI, D. P. Metodologia SSM e sua aplicação na intervenção organizacional em uma empresa de propaganda. 10 Congresso Brasileiro de Sistemas, 2005. Disponível em: < http://legacy.unifacef.com.br/quartocbs/arquivos/08.pdf >. Acesso em: 20 mai. 2015.

CHECKLAND, P. Soft Systems Methodology: A Thirty Year Retrospective (a). Systems Research and Behavioral Science, v. 17, n. S1, p. S11-S58, 2000.

DOMINGUES, C. R.; BUENO, J. M. Impacto Da Resistência À Mudança Na Implantação Do Balanced Score Card. 2004. Revista da FAE. Disponível em:

<http://www.unifae.br/publicacoes/pdf/art_cie/art_21.pdf >. Acesso em: 10 mar. 2014.

DRUCKER, P. Desafios Gerenciais para o Século XXI. São Paulo: Pioneira, 1999.

FEROLLA, L. M. Processos colaborativos na gestão pública: estudo das relações estabelecidas no contexto do Programa Nacional de Educação do Campo. Dissertação de Mestrado. Departamento de Administração, Universidade de São Paulo, Ribeirão Preto, 2013. 204 p. 
FLEURY, M. T. L. Organizational culture and the renewal of competences. BAR - Brasilian Administration Review, v. 6, n. 1, p. 14, 2009.

FREITAS, M. E. Cultura organizacional: Grandes temas em debate. RAE-Revista de Administração de Empresas, v. 31, n. 3, p. 10, 1991.

FREZATTI, F.; RELVAS, T. R. S.; JUNQUEIRA, E. BSC e a Estrutura de Atributos da Contabilidade Gerencial: uma Análise no Ambiente Brasileiro. RAE-Revista de Administração de Empresas, v. 50, n. 2, p. 12, 2010.

FURLAN JR, V.; CARDOS, A. A.; CHAVES, C. A. Estudo de Aderência da implantação de um programa de melhoria contínua às práticas de gerenciamento de projeto. Revista de Ciências Exatas, v. 16, n. 1, p. 65-71, 2010. Disponível em: <http://periodicos.unitau.br/ojs2.2/index.php/exatas/article/view/1278/857>. Acesso em: 10 mai. 2015.

GIL, A. C. Como elaborar projetos de pesquisas. São Paulo: Atlas, 2002.

KAPLAN, R. S.; NORTON, D. P. A estratégia em ação: balanced scorecard. São Paulo: Campus, 1997.

KAPLAN, R. S.; NORTON, D. P. Mapas estratégicos: convertendo ativos intangíveis em resultados tangíveis. Rio de Janeiro: Campus, 2004.

LIKER, J. K.; DAVID, M. O Modelo Toyota Manual de Aplicacao. Porto Alegre: Bookman Companhia Ed., 2005.

MINTZBERG, H.; AHLSTRAND, B.; LAMPEL, J. Safari da estratégia. Porto Alegre: Bookman Companhia Ed., 2010.

MORALES, H. N.; GONZALEZ, J. G.; MENDOZA, A. T. Empowerment As A Culture And A Strategy To Strengthen The Activities Of Research And Innovation: Proposal Of A Methodology. European Scientific Journal, v. 1, n. 1, p. 13, 2013.

NEIVA, E. R.; PAZ, M. G. T. Percepção de mudança individual e organizacional: o papel das atitudes, dos valores, do poder e da capacidade organizacional. Revista de Administração, v. 47, n. 1, p. 16, 2012.

NEIS, D.; PEREIRA, M. F. As origens do campo da estratégia: contribuições de Igor Ansoff e Henry Mintzberg. Revista Eletrônica de Estratégia \& Negócios, v. 8, n. 2, p. 207 - 240, jan. 2016.

OLIVEIRA, N. G., TERRA, L. A. A. Prevalência das escolas de pensamento estratégico na implantação de sistemas ERP em concessionárias de veículos. Revista Eletrônica de Estratégia \& Negócios, 6, mai. 2013. Disponível em: <http://portaldeperiodicos.unisul.br/index.php/EeN/article/view/940/1094>. Acesso em: 27 nov. 2014.

PORTER, M. Estrategia Competitiva. Rio de Janeiro: Campus, 2004. 
POWELL, T. C. Total Quality Management as Competitive Advantage A Review and Empirical Study. Strategic Management Journal, v. 16, n. 1, p. 15-37, 1995.

ROSE, J. Soft Systems Methodology as a Social Science Research Tool. Systems Research and Behavioral Science, v. 14, n. 4, p. 249-258, 1997.

SANTOS, J. V.; GONÇALVES, G. A cultura organizacional: O impacto visível de uma dimensão invisível. Psico, v. 41, n. 3, p. 6, 2010.

SHIRI, A.; SOLTANI, M. D.; ABADI, E. G. S. The effect of strategy of organizational assessment upon organizational empowerment in Kerman's administrative apparatuses. Journal of Basic and Applied Scientific Research, v. 3, n. 8, p. 387-393, 2013. Disponível em: < http://www.textroad.com/pdf/JBASR/J.\%20Basic.\%20Appl.\%20Sci.\%20Res.,\%203(8)387393,\%202013.pdf >. Acesso em: 10 mai. 2014.

SILVA, L. C. O Balanced Scorecard e o Processo Estratégico. Caderno de Pesquisas em Administração, v. 10, n. 4, p. 52003.

SKINNER, B. F. Sobre o behaviorismo. São Paulo: Cultrix, 1982.

SLACK, N; CHAMBERS, S; JOHNSTON, R. Administração da Produção. Atlas, 2009.

SOBRAL, F.; PECl, A. Administração: teoria e prática no contexto brasileiro. São Paulo: Prentice Hall Brasil, 2008.

SPESSATTO, G.; BEUREN, I. M. Análise das diferenças na implantação do balanced scorecard nas maiores empresas da região sul do Brasil. Gestão \& Produção, v. 20, n. 2, p. 17, 2013.

TERRA, L. A. A.; PASSADOR, J. L. Symbiotic Dynamic: The Strategic Problem from the Perspective of Complexity. Systems Research and Behavioral Science, DO I: 10.1002/sres.2379, 2015.

WOOD JR, T.; CURADO, I. B.; CAMPOS, H. M. Vencendo a crise: Mudança organizacional na Rhodia Farma. RAE-Revista de Administração de Empresas, v. 34, n. 5, p. 62-79, 1994. 